\title{
Performance evaluation of a new selective plugging agent system for water producing gas well
}

\author{
Jian Yan ${ }^{1, \text { a Jiangshuai Wang }}{ }^{1, b}$ Haiyan $L^{2, c}$ Zhuanhong $\mathrm{Li}^{2, \mathrm{~d}}$ \\ ${ }^{1}$ Xi'an Shiyou University, Xi'an, 710065, China \\ ${ }^{2}$ Oil and Gas Technology Institute of Changqing Oilfield, Xi'an, 710065, China

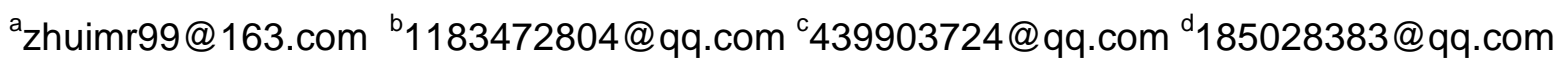

Keywords: selective plugging agent; water producing gas well; performance evaluation.

Abstract. To evaluate the performances of a new selective plugging agent system, the lab experiments are carried out, it can be concluded that: The plugging agent system has the ability of selective plugging for water and gas; with the increase of the water saturation, the plugging rate of both water and gas are increased; under the same water saturation, the larger the permeability is, the lower the plugging rate is; with the increase of the water saturation, the entering depth of the plugging agent is reduced, but its effect is less than the permeability. The studying results provide theoretical reference for the selective plugging of water of water production gas well.

\section{Introduction}

As part of the gas reservoir contains formation water, in the production process, gas wells will have varying degrees of water production. For these wells, if the water can be solved by using plugging water technique directly, can not only reduce processing costs, increase economic benefits, but also to avoid the treatment of produced water after water drainage, and reduce the pollution of the environment, controlling the formation sand production, pipeline corrosion and scaling phenomenon. In particular, it is more necessary to study the water plugging technology for the treatment of the water producing gas wells with high cost or the field without drainage gas recovery technology. But the water plugging technology in gas wells may be more trouble than well, we should study the special water plugging agent and process technology according to the specific characteristics of the gas well, and can not completely copy the oil well water plugging agent and process technology.

\section{Materials and plugging agent component}

The main polymer: polyacrylamide (PAM); crosslinking agent: urotropine and organic chromium; sodium sulfite; ammonium chloride; tap water; artificial cores.

Plugging agent formulation: $0.7 \%$ polyacrylamide (PAM), $0.8 \%$ organic chrome crosslinking agent, $0.35 \%$ urotropine, $0.19 \%$ sodium sulfite, $0.21 \%$ chloride ammonium.

Range of rock permeability: $71 \times 10^{-3} \mu \mathrm{m}^{2} \sim 385 \times 10^{-3} \mu \mathrm{m}^{2}$.

\section{Plugging mechanism}

Polyacrylamide is a typical selective plugging system. Here, sodium sulfite and ammonium chloride are added into the system. When components in the stratigraphic pores are heated to $80^{\circ} \mathrm{C}$, ammonium chloride and sodium nitrite will react and generate nitrogen, which makes the volume of the origin components increase, and firmly remain in the pores to have a good effect of water isolation. 


\section{Plugging rate in different water saturation}

Testing procedures:

Take two dry artificial cores, one of which is tested gas phase permeability, another is tasted the permeability of water phase. Then, make the wet core dry, at this time, the water saturation of two cores are about $0 \%$;

Displacement the plugging agent 1PV in low pressure and low injection rate;

Keep the cores under the conditions of $80^{\circ} \mathrm{C}$ for 13 hours, to make the plugging agent fully adsorbed on the surface of the core pores and is formed gel in pores;

Drive the two cores by gas at $80^{\circ} \mathrm{C}$ and under a certain pressure, test the gas permeability of the gas phase after plugging, and calculate the rate of gas blockage;

Drive the two cores by gas at $80^{\circ} \mathrm{C}$ and under a certain pressure, test water permeability after plugging, and calculate the rate of water blocking.

Permeability determination under different water saturation: take two cores with similar permeability, drive cores with water to form a certain water saturation, measure the permeability of water phase and the gas phase permeability, then repeat the above steps.

The testing results are shown in figure 1. It can be seen from the graph that with the increase of the water saturation, the plugging rate of both water and gas are increased. The lower the reservoir water saturation is, the lower the blocking ability of plugging agent for gas and water, this is because in low water saturation of reservoir, most of the pore volume is filling gas, water only adsorbs on rock surface, so the adsorption capacity of plugging agent in the gas reservoir is low. But the plugging agent of cationic polymer is linear in water, high molecular are intertwined with each other, blocking the flow of water, the polymer contact with gas, and the long chain of the polymer can not stretch, unable to form a continuous space network structure, so the plugging rate of gas is small. In the gas reservoir with higher water saturation, the water is the continuous phase and the gas is the dispersed phase, a part the injected plugging agent can be adsorbed on the surface of the rock, the adsorption of cationic polymers in porous rocks is irreversible, polymer on solid surface can form many adsorption sites, even if each of the keys are very weak, but the sum of bond will have a strong adsorption force, so all the keys are unlikely broken at the same time. Therefore, for the gas reservoirs with higher water saturation, the water plugging rate is higher.

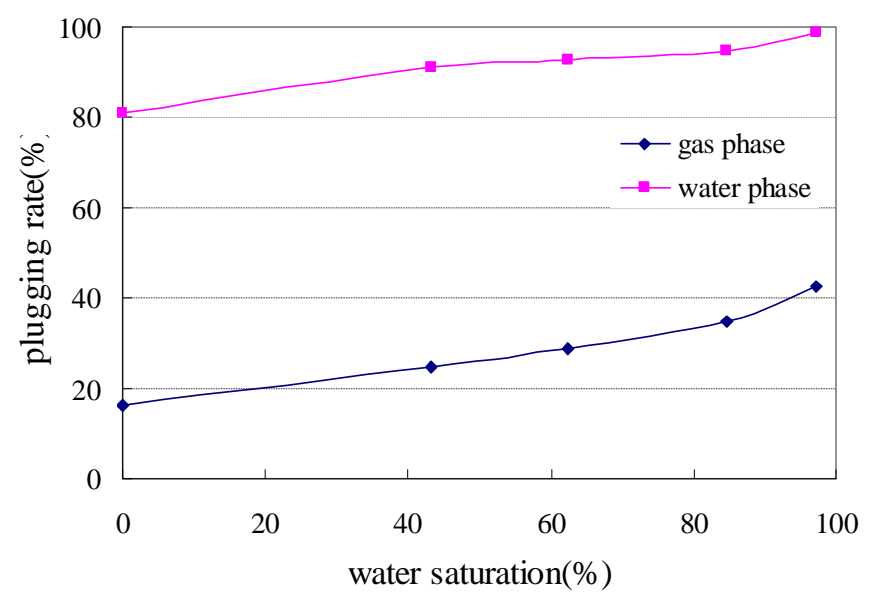

Fig.1 Relationship curve of the plugging rate via water saturation

\section{Plugging rate of cores with different permeability}

Using the cores with different permeability, the effect of permeability on plugging rate is tested. The plugging agent system is the same with above. Only the test condition is under the same water saturation about $80 \%$.

The testing results are shown in figure 2. It can be seen that with the increase of formation permeability, water plugging rate and blocking rate of plugging agent are decreased, which is due to 
the low content blocking agent in order to reduce the blockage of the gas in the low permeability layer and the larger the permeability is, the larger the pore throat diameter is, the plugging efficiency is reduced. That is, under the same water saturation, the larger the permeability is, the lower the plugging rate is. For the higher permeability reservoirs, to improve the plugging rate, other higher efficiency plugging technologies should be adopted.

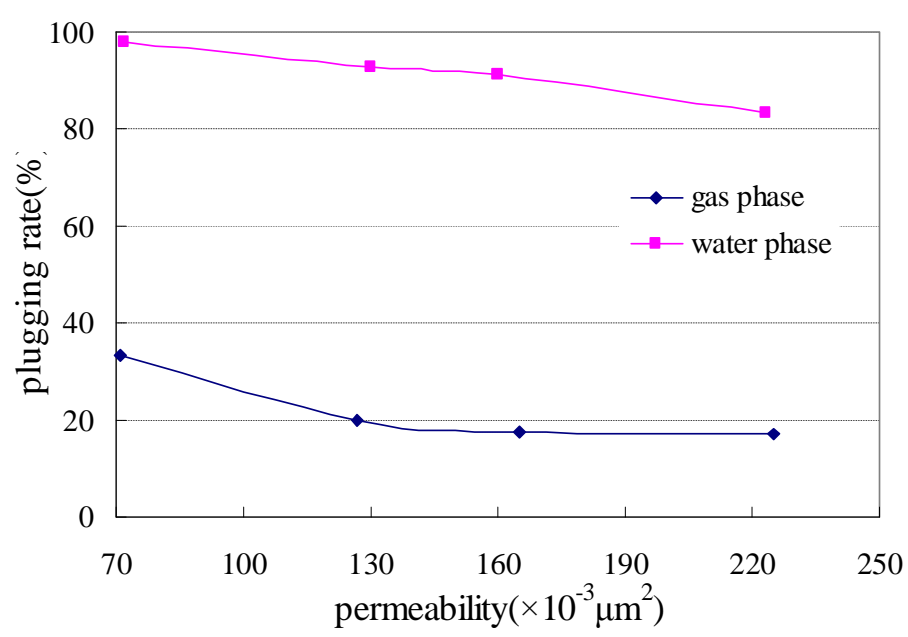

Fig.2 Relationship curve of the plugging rate via permeability

\section{Entering depth of plugging agent into the cores}

The entering depth of plugging agent is studied for both permeability and water saturation.

The testing procedures for the first situation are as following:

Select four cores with different permeability and parallel connect them;

Displacement the plugging agent under low pressure and low injection rate;

Stop the injection when one of them flows out the plugging agent from the outlet;

Cut the other three cores to measure the entering depth of the plugging agent.

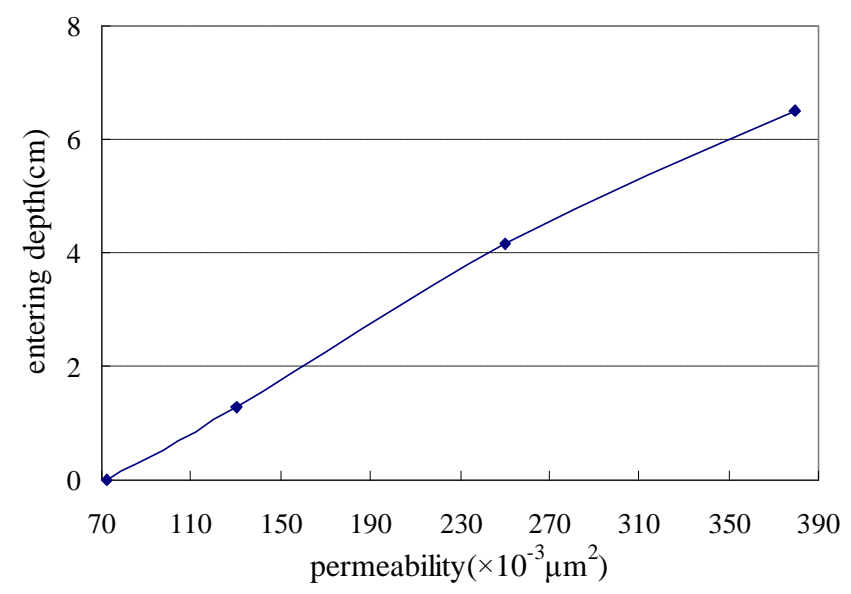

Fig.3 Relationship curve of the entering depth via permeability

The test results are shown in figure 3. It indicates that the size of permeability determines the depth of plugging agent into cores, the plugging agent first enter the high permeability core, the higher the core permeability is, the greater the entering depth of the selection plugging agent. Combined with the above results, it should also know that the deeper the entering depth does not mean the larger plugging rate for both water and gas.

The testing procedures for the second situation are similar to that of studying the plugging rate in different water saturation. After those procedures, cut the cores to measure the depth of plugging agent. 
The testing results are shown in figure 4 . With the increase of the water saturation, the entering depth of the plugging agent is reduced, but its effect is less than the permeability. Therefore, the depth of the plugging agent is mainly affected by the permeability of the core.

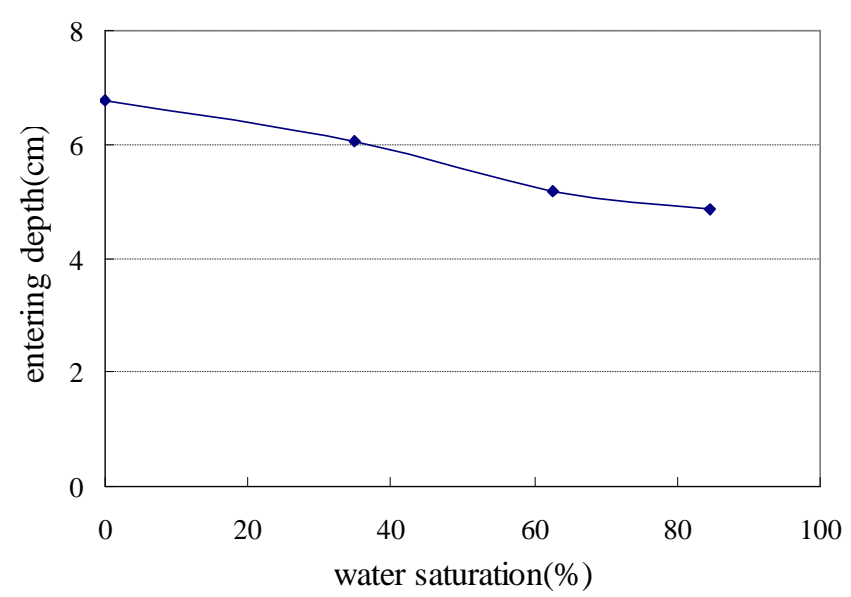

Fig.4 Relationship curve of the entering depth via water saturation

\section{Conclusion}

By the lab experiments, it can be concluded that:

The plugging agent system has the ability of selective plugging for water and gas.

With the increase of the water saturation, the plugging rate of both water and gas are increased.

Under the same water saturation, the larger the permeability is, the lower the plugging rate is.

With the increase of the water saturation, the entering depth of the plugging agent is reduced, but its effect is less than the permeability.

\section{Acknowledgments}

We would like to thank College of Petroleum Engineering in Xi'an Shiyou University for providing experimental conditions. In addition, it is supported by national natural science fund item "Influence mechanism and correction of gas \& water relative permeability curve in tight gas reservoir" (51404197) and innovation and entrepreneurship training program for college students "Selective plugging agent system optimization and process improvement of gas well in low permeability gas reservoir".

\section{References}

[1] SYDANSK R D, SERIGHT R S. When and where relative permeability modification water shutoff treatments can be successfully applied[R]. SPE99371, 2006

[2] TONG Zhi-neng. Performance and Evaluation of a New Type of Selective Blocking Agent [J]. Science Technology and Engineering. 2010,5(10):1231-1232

[3] BORODNEG,VAKHROUSHEV P E, STOLYAROV S M, et al. Application of relative permeability rnodifiers to control water cut following hydraulic; fracturing in western Siberia oil fields - Russian case-history study[J]. SPE Production\& Operations. 2008, 23(3): 411-418 